\title{
A Heuristic for Decomposition in Multilevel Logic Optimization
}

\author{
Vinaya Kumar Singh and Ajit A. Diwan
}

\begin{abstract}
In this paper, we propose a new heuristic to find common subexpressions of given Boolean functions based on Shannon-type factoring. This heuristic limits the search space of finding common subexpressions considerably by applying a top-down approach. In this top-down approach, synthesis of a Boolean network flows from the primary outputs to the primary inputs. The common subexpressions and their complements in $\boldsymbol{N}$ variables are extracted before common subexpressions and their complements in $(N-1)$ variables. This decomposition of the network depends upon a permutation of Boolean variables and has a polynomial complexity for restricted extraction of complements.

A multilevel logic optimization system, MULTI, has been implemented using this heuristic. Good results on several benchmark circuits show its effectiveness.
\end{abstract}

\section{INTRODUCTION}

$\mathrm{C}$ YOMMON subexpression extraction and factoring are two basic optimization methods for technology-independent multilevel logic optimization [1]. In many multilevel logic synthesis systems [2], [6], algorithms of [1] have been used for multilevel logic optimization. These algorithms are based on the notion of a kernel which is a subexpression that cannot be factored fully. Common subexpressions are computed by finding common kernels and kernel intersections. Since there can be an exponential number of kernels in the number of Boolean variables, these operations take an impractical amount of time for many circuits. Because of this, in optimization of large circuits, these operations are restricted to level-0 kernels only. This problem is particularly evident in the case of symmetric Boolean functions [5].

The approach of [1] with level-0 kernels can be viewed as a bottom-up approach to synthesizing given Boolean functions. In the first step, common kernels of primary inputs are found. There kernels are used recursively to synthesize higher level kernels and finally the primary outputs. As there will be too many kernels at low levels for large circuits, getting a good kernel is more costly and difficult. This limitation of a kernel-based algorithm has been addressed in [8], in which only two literal single cubes and double cube divisors and their complements are

Manuscript received March 25, 1993; revised July 26, 1993. V. K. Singh is with Silicon Automation Systems Lid., Indira Nagar, Bangalore, India.

A. A. Diwan is with the Department of Computer Science and Engineering, Indian Institute of Technology, Powai, Bombay-76, India. IEEE Log Number 9213088 considered. This speeds up the common subexpression extraction process considerably. In this paper, we have proposed a new heuristic which handles this problem efficiently.

The proposed heuristic is a top-down approach to synthesizing a multilevel logic and starts by finding common subexpressions equivalent to higher-level kernels. In this heuristic, the problem of finding common subexpressions of a given circuit has been transformed into the problem of finding a good ordering on the Boolean variables of the circuit. It has a polynomial time complexity in the size of inputs if finding complements is restricted to functions of a constant number of variables.

The system MULTI uses this heuristic to find global common subexpressions. A given Boolean network is globally optimized when this heuristic is used. Local transformation of misII [2] are used on the resultant network. This system has been found to give excellent results for symmetric function and large circuits.

\section{Definitions}

This section contains definitions which have been used to describe the algorithms. The representation of a circuit in the form of Boolean expressions has been taken from [2].

A variable or a Boolean variable is a symbol representing a signal which can take the value 0 or 1 . A variable $v$ in its complemented (i.e., $v^{\prime}$ ) or uncomplemented (i.e., $v$ ) form is a literal.

A cube is a set of literals such that if literal $l$ is present, then literal $l^{\prime}$ is not present. A cube represents the conjunction of its literals. For example, set $\{a, b\}$ is a cube representing conjunction $a * b$. The Boolean functions 0 and 1 are represented by cubes 0 and 1 , respectively.

An expression or subexpression is a set of cubes. An expression represents a disjunction of cubes. For example, set $\{\{a, b\},\{b, c\}\}$ represents disjunction of cube $a$ $* b$ and $b * c$ (i.e., $a * b+b * c$ ). The symbolic representation (e.g., $a * b+b * c$ ) will be used interchangeably for the set representation (e.g., $\{\{a, b\},\{b, c\}\}$ ). The size of an expression $e$ is defined as the number of cubes in $e$. For example, the size of expression $a * b+$ $b * c$ is 2 .

The support of an expression is a set of variables used in the expression. The support of expression $f$ is set $V, V$ $=\operatorname{support}(f)=\left\{v \mid v \in C\right.$ or $v^{\prime} \in C$ and cube $\left.C \in f\right\}$.

$A$ factored expression is defined as follows. 
1) A literal is a factored expression.

2) A conjunction of factored expressions is a factored expression.

3) A disjunction of factored expressions is a factored expresssion.

For example, $a *\left(b^{\prime} * c^{\prime}+b * c\right)+a^{\prime} *\left(b^{\prime} * c+b *\right.$ $\left.c^{\prime}\right)$ is a factored expression.

The literal factor of an expression $f$ with respect to a literal $l$ is a set of cubes of $f$ containing $l$ with $l$ deleted: i.e., $L F(f, l)=\{C-\{l\} \mid$ cube $C \in f$ and $C \cap\{l\} \neq$ $\phi\}$.

The variable factor of expression $f$ with respect to a variable $v$ is a set of expressions, defined as

$$
V F(f, v)=\left\{h_{1}, h_{2}, r\right\}
$$

where

$$
\begin{aligned}
h_{1}= & L F(f, v) \\
h_{2}= & L F\left(f, v^{\prime}\right) \\
r= & \left\{C \mid \text { cube } C \in f \text { and } C \cap\{v\}=\phi \text { and } C \cap\left\{v^{\prime}\right\}\right. \\
& =\phi\} .
\end{aligned}
$$

For example, the variable factor of expression $a * b * c$ $+a * b^{\prime} * c^{\prime}+a^{\prime} * b * c^{\prime}+a^{\prime} * b^{\prime} * c$ with respect to variable $a$ is set $\left\{b * c+b^{\prime} * c^{\prime}, b * c^{\prime}+b^{\prime} * c\right\}$.

A node is defined by the Boolean equation $f=e$, where $f$ is the output signal of the node and expression $e$ represents a Boolean relation between $f$ and support $(e)$.

A Boolean network [2] represents a cycle-free combinational multilevel circuit. It is represented by a set of nodes. We define a Boolean network to be a set of Boolean equations.

The cost of a Boolean equation $f=e$ is defined as the number of literals in expression $e$. For example, the cost of Boolean equation $f=a * b * c+a * b^{\prime} * c^{\prime}+a^{\prime} * b$ $* c^{\prime}+a^{\prime} * b^{\prime} * c$ is 12 .

The cost of a Boolean network is defined as the sum of costs of each node of the network. For example, the cost of Boolean network $N=\left\{f_{1}, f_{1}\right\}$

where

$$
\begin{aligned}
f_{1}= & a * b * c+a * b^{\prime} * c^{\prime}+a^{\prime} * b * c^{\prime} \\
& +a^{\prime} * b^{\prime} * c
\end{aligned}
$$

and

$$
\begin{aligned}
f_{2}= & a^{\prime} * b^{\prime} * c^{\prime}+a * b * c^{\prime}+a * b^{\prime} * c \\
& +a^{\prime} * b * c \text { is } \\
\operatorname{cost}(N)= & \operatorname{cost}\left(f_{1}\right)+\operatorname{cost}\left(f_{2}\right)=12+12=24 .
\end{aligned}
$$

\section{Permutation Decomposition}

Permutation decomposition is a fast heuristic to extract common subexpressions of a Boolean network. In permutation decomposition, a Boolean network $N$ is transformed into an equivalent Boolean network $N^{\prime}$, such that all common subexpressions in variables $V_{i}, \cdots, V_{n}$ for $1<i<n$ and $n=|V|$ for a given permutation $V$ of

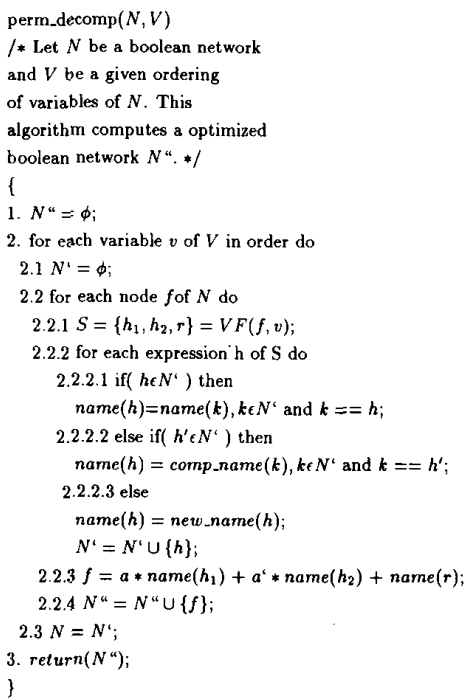

Fig. 1. Algorithm perm_decomp.

variables $N$ are expressed as intermediate nodes, and these intermediate nodes are substituted in the nodes of $N$.

The algorithm for permutation decomposition is given in Fig. 1. The functions used are as follows:

name (): the name of a node is the output signal associated with it, whereas the name of an expression is the output signal of the node with this expression.

comp_name( ): the comp_name of a node is the complement of the output signal of the node.

Example 1: Let

$$
\begin{aligned}
f_{1}= & a * b * c+a * b^{\prime} * c^{\prime}+a^{\prime} * b * c^{\prime} \\
& +a^{\prime} * b^{\prime} * c \\
f_{2}= & a^{\prime} * b^{\prime} * c^{\prime}+a * b * c^{\prime}+a * b^{\prime} * c \\
& +a^{\prime} * b * c
\end{aligned}
$$

be a Boolean network and $V=\{a, b, c\}$ a permutation. The Boolean network obtained after permutation decomposition is

$$
\begin{aligned}
f_{1} & =a *[0]+a^{\prime} *[0]^{\prime} \\
f_{2} & =a *[0]^{\prime}+a^{\prime} *[0] \\
{[0] } & =b * c+b^{\prime} * c^{\prime} .
\end{aligned}
$$

Step (2.2.2.2) in the algorithm perm_decom shown in Fig. 1 requires determining whether two expressions are a complement of each other. This problem is equivalent to the problem of finding a dissatisfying assignment to a $D N F$ formula, which is NP-complete [4]. In this algorithm, this step is restricted to the expressions of a fixed number of variables.

The optimization done by permutation decomposition depends upon the given permutation of the Boolean vari- 
ables. Exploring all possible permutations is a costly operation. We have used a greedy algorithm to decide upon a good permutation.

If an expression is symmetric, then the permutation decomposition on any permutation of variables is the best decomposition. In this case, common subexpressions of all possible weights [5] are identified.

The optimization by permutation decomposition can be viewed as a top-down decomposition, in which common expressions with more variables are extracted first. These common expressions are decomposed recursively until common expressions in two inputs are obtained.

\section{Multilevel Logic Optimization}

The multilevel logic optimization algorithm based on permutation decomposition is given in Fig. 2. The functions used in the algorithm are as follows.

simplify( ): simplify is misII transformation which makes each node of the network irredundant with single cubic containment.

partition( ): partition splits a network $N$ into a set of networks $\left\{N_{1}, N_{2}, \cdots, N_{n}\right\}$, such that at most one variable is shared between $N_{i}$ and $N_{j}, i \neq j$ and $1 \leq i, j$ $\leq n$. This speeds up optimization specifically for large multilevel combinational circuits.

select_perm( ): select_perm uses a greedy algorithm to select a good permutation of variables of the Boolean network.

factor( ): factor is the same as the literal factoring algorithm of [2]. Kernel factoring [6] is not required since all good common subexpressions have already been found.

Example 2: Let

$$
\begin{aligned}
& f_{1}=a * c+a * d+a * e \\
& f_{2}=b * c+b * d+b * e \\
& f_{3}=a * c+b * c \quad f_{4}=a * d+b * d
\end{aligned}
$$

be a Boolean network. The permutation $V=\{a, b, c, d$, $e\}$ is selected by select_perm. The optimized Boolean network obtained after permutation decomposition on this permutation is

$$
\begin{array}{rlrl}
f_{1} & =a *[0] & & f_{2}=b *[0] \\
f_{3} & =a * c+b * c & f_{4}=a * d+b * d \\
{[0]} & =c+d+e . & &
\end{array}
$$

The optimization steps are repeated again since the cost of the new network has improved. The final optimized Boolean network is

$$
\begin{array}{rlrl}
f_{1} & =a *[0] \quad f_{2} & =b *[0] \\
f_{3} & =c *[1] \quad f_{4} & =d *[1] \\
{[0]} & =c+d+e & {[1]} & =a+b .
\end{array}
$$

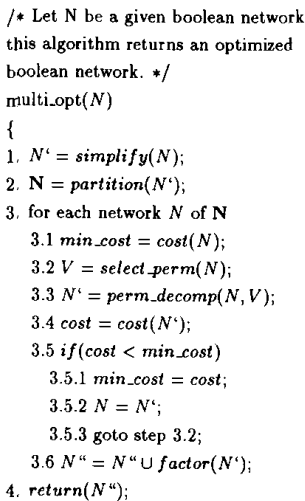

Fig. 2. Algorithm multi_opt.

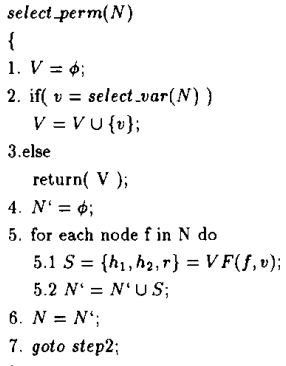

Fig. 3. Algorithm select perm.

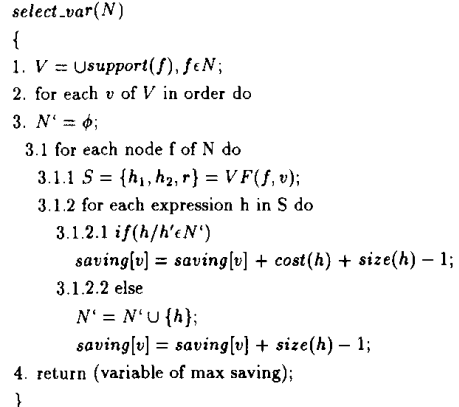

Fig. 4. Algorithm select_var.

\section{A. Selection of Permutation}

A greedy algorithm to find a good permutation of a Boolean network is a given in Fig. 3. A variable which gives maximum saving in literal count is selected by select var (Fig. 4) when variable factor $V F$ is applied to each node of the given Boolean network. The nodes of the network are decomposed using $V F$ for the selected variable and a new network is obtained. This takes into consideration the changed savings of remaining variables. The next best variable is selected by select_var and the steps are repeated similarly for the remaining variables. 
TABLE I

\begin{tabular}{|c|c|c|c|c|c|c|c|}
\hline \multirow{2}{*}{$\begin{array}{l}\text { BENCH-MARK } \\
\text { CIRCUIT }\end{array}$} & \multirow{2}{*}{\begin{tabular}{c|} 
INITIALL \\
\# LITERALS
\end{tabular}} & \multicolumn{3}{|c|}{ \# LITERALS IN SOP } & \multicolumn{3}{|c|}{ CPU TIME* } \\
\hline & & multi & sis-1.1.1* & sis $1.1^{* * *}$ & multi & sis-1.1** & $\operatorname{sis} 1.1^{* * *}$ \\
\hline tst & 2028 & 163 & 202 & 217 & 29.7 & 102.0 & 239.2 \\
\hline sym10 & 8370 & 105 & 195 & 248 & 58.4 & 710.3 & 147.3 \\
\hline sym9 & 3780 & 81 & 275 & 229 & 17.2 & 272.5 & 28.5 \\
\hline alu & 6150 & 368 & 401 & 461 & 95.5 & 482 & 405.2 \\
\hline 8 bitadder & 21692 & 104 & 136 & 215 & 1301.5 & 992.4 & 750.4 \\
\hline
\end{tabular}

*CPU time is in seconds on a Sun SPARC IPC. **sis-1.1 with rugged script. $* * *$ sis-1.1 with Boolean script

TABLE II

\begin{tabular}{|c|c|c|c|c|c|c|c|}
\hline \multirow{2}{*}{$\begin{array}{c}\text { BENCH-MARK } \\
\text { CIRCUIT } \\
\end{array}$} & \multirow{2}{*}{$\begin{array}{c}\text { INITIAL } \\
\text { \# LITERALS }\end{array}$} & \multicolumn{3}{|c|}{ \# LITERALS IN SOP } & \multicolumn{3}{|c|}{ CPU TIME* } \\
\hline & & multi & sis-1.1** & sis1.1*** & multi & sis-1.1. $1^{* *}$ & sisl.1. \\
\hline $5 x p 1$ & 303 & 108 & 147 & 168 & 5.8 & 13.2 & 6.7 \\
\hline apex1 & 9133 & 1722 & 1676 & 1822 & 1041 & 1033.3 & 2273.6 \\
\hline apex2 & 14871 & 456 & - & - & 328.4 & - & - \\
\hline apex3 & 8397 & 1961 & - & 2152 & 870 & - & 739 \\
\hline alupla & 211 & 160 & 144 & 169 & 11.8 & 10.4 & 5.6 \\
\hline $551 \mathrm{~m}$ & 327 & 127 & 109 & 172 & 7 & 10.0 & 7 \\
\hline sao2 & 493 & 171 & 192 & 223 & 22.1 & 24.7 & 7 \\
\hline rd73 & 843 & 135 & 73 & 112 & 11.2 & 12.3 & 17.5 \\
\hline
\end{tabular}

*CPU time is in seconds on a Sun SPARC IPC.

***is-1.1 with rugged script.

$* * *$ sis-1.1 with Boolean script.

-System ran out of space (20 MB main memory and $200 \mathrm{MB}$ swap space).

TABLE III

\begin{tabular}{|l|r|r|r|r|r|}
\hline \multirow{2}{*}{$\begin{array}{c}\text { BENCH-MARK } \\
\text { CIRCUIT }\end{array}$} & \multicolumn{1}{c|}{$\begin{array}{c}\text { INITIAL } \\
\text { \# LITERALS }\end{array}$} & \multicolumn{2}{|c|}{ \# LITERALS IN SOP } & \multicolumn{2}{|c|}{ CPU TIME** } \\
\cline { 3 - 6 } & multi & \multicolumn{1}{c|}{ sis-1.1 } & & multi & sis-1.1** \\
\hline c2670 & 2075 & 815 & 995 & 28 & 71 \\
\hline c3540 & 2936 & 1421 & 2319 & 55 & 309 \\
\hline c5315 & 4386 & 1970 & 2007 & 182 & 159 \\
\hline c6288 & 4800 & 3328 & 4619 & 80 & 728 \\
\hline c7552 & 6144 & 2501 & 2838 & 119 & 638 \\
\hline
\end{tabular}

*CPU time is in seconds on a Sun SPARC IPC.

**sis-1. 1 with Boolean script.

\section{Experimental Results}

The results of our system MULTI on ISCAS [3] and MCNC benchmarks are presented in Tables I, II, and III. The results have been compared with the best scripts of sis1.1, which has a fast common subexpression extraction algorithm [8] in misII framework.

Table I shows the results on some large circuits which have a large number of kernels. The number of kernels for these circuits is shown in Table IV.

The benchmarks sym9, sym10, and alu are ISCAS and 8 bitadder is an 8 bit adder in which outputs and carry out are expressed as the sum of products of inputs and carry in.

The circuits $t s t$, sym 10 , and sym 9 are symmetric functions and 8bitadder and alu have good higher level kernels. The excellent results for these circuits can be explained by the fact that being a top-down approach, by our system is able to extract higher level common expressions and their complements efficiently. This system synthesizes ripple carry adder for the circuit 8bitadder.
TABLE IV

\begin{tabular}{|l|r|r|r|r|}
\hline BENCH-MARKS & PI & PO & \# LITERALS & \# KERNELS \\
\hline tst & 3 & 3 & 2028 & 1478 \\
\hline sym10 & 10 & 1 & 8370 & 53745 \\
\hline sym9 & 9 & 1 & 3780 & 17761 \\
\hline alu & 14 & 3 & 6150 & 51468 \\
\hline 8bitadder & 17 & 9 & 21092 & 63079 \\
\hline
\end{tabular}

Table II shows results on MCNC benchmarks. For the circuits $5 x p 1$, apex 2 , and apex3, MULTI produces smaller circuits as compared to the best circuits produced by sis1.1 .

Table III presents the results on large multilevel benchmarks of [3]. These circuits have a small number of kernels but have many partitions. Reduction in the circuit size by partitioning results in the fast optimization of these circuits.

\section{CONCLUSION}

Generation of good common subexpressions is very important for optimizing the global structure of a Boolean network, but it is a computationally hard problem. The permutation decomposition which we have proposed models this problem effectively and restricts the search space considerably. The optimization by this decomposition algorithm depends upon the permutation of Boolean variables on which the decomposition is done. An effective greedy algorithm for finding a permutation of a Boolean variable is discussed.

Future work will involve exploring the following issues for possible improvements.

1) A better heuristic for deciding upon the permutation of variables.

2) Restricting a permutation decomposition to a fixed depth and searching for another permutation.

3) Use of Boolean properties.

4) A mixed-top-down and bottom-up approach.

\section{REFERENCES}

[1] R. K. Brayton and C. McMullen, "The decomposition and factorization of Boolean expressions," in Proc. Int. Symp. Circuits Syst. (ISCAS-82), May 1982, pp. 49-54.

[2] R. K. Brayton et al., "MIS: A multiple-level logic optimization system," IEEE Trans. Comput.-Aided Design, vol. CAD-6, pp. 10621081, Nov. 1987

[3] A. J. DeGeus, "Logic synthesis and optimization benchmark for the 1986 design automation conference," in Proc. 23rd IEEE Design Automation Conf., 1986, p. 78.

[4] M. R. Garey and D. S. Johnson, Computers and Intractability-A Guide to Theory of NP-Completeness. San Francisco: Freeman, 1979.

[5] B. G. Kim and D. L. Dietmeyer, "Multilevel logic synthesis of symmetric switching functions," IEEE Trans. Comput-Aided Design, vol. 10, pp. 436-446, Apr. 1991.

[6] G. De Micheli et al., Design Systems for VLSI Circuits. Dordrecht. The Netherlands: Martinus Nijhoff. 1987.

[7] Y. Nakamura et al.. "Area optimization for large circuits by partial collapsing," in Proc. Int. Symp. Logic Synthesis and Microprocessor Architecture. July 1992, pp. 28-33.

[8] J. Rajski and J. Vasudevamurthy. "The testability-preserving concurrent decomposition and factorization of Boolean expressions," IEEE Trans. Comput.-Aided Design, vol. 11, pp. 778-793, June 1992. 


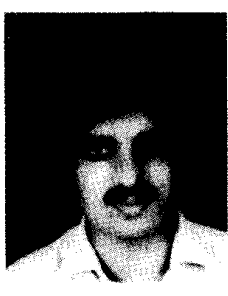

Vinaya Kumar Singh received the B.E. degree in computer science from the Regional Engineering College Allahabad, India, in 1989 and the M. Tech. degree in computer science from the Indian Institute of Technology, Bombay, in 1992

Since January 1992, he has been working at Silicon Automation Systems, Bangalore, India, where he is involved in the design and development of Verilog synthesis tools. His research interests are high-level synthesis, logic synthesis, and algorithms.

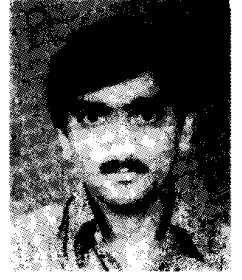

Ajit A. Diwan received the B.Tech. degree in electrical engineering from the Indian Institute of Technology, Bombay, in 1983, and a Ph.D. degree in computer science from Bombay University in 1989 .

Since 1988, he has been working at I.I.T Bombay, where he is currently an Assistant Professor. His research interests are in algorithms, combinatorics, and graph theory, with applications to VLSI design. 